\title{
Discrete shanker distribution and its derived distributions
}

\section{Introduction}

One parameter continuous Shanker distribution introduced by Shanker $(2015$ b) with parameter $\theta$ is defined by its probability density function (pdf).

$$
f(x: \theta)=\frac{\theta^{2}}{\theta^{2}+1}(\theta+x) e^{-\theta x} \cdot x>0 . \theta>0 .
$$

\section{Discretization of continuous distribution}

Discretization of continuous distribution can be done using different methodologies. In this paper we deal with the derivation of a new discrete distribution which takes values in $\{0,1, \ldots\}$. This new distribution is generated by discretizing the continuous survival function of the Shanker distribution, which is may be obtained as

$$
\begin{gathered}
S(x)=\int_{x}^{\infty} f(x: \theta) d x \\
=\frac{\theta^{2}+1+\theta x}{\theta^{2}+1} e^{-\theta x}, x>0 . \theta>0 . \\
S(x+1)=\frac{\theta^{2}+1+\theta(x+1)}{\theta^{2}+1} e^{-\theta(x+1)}, x>0 . \theta>0 .
\end{gathered}
$$

The probability mass function (pmf) of discrete Shanker distribution may be obtained as

$$
\begin{gathered}
P(X=x)=S(x)-S(x+1) \\
=\frac{\left(\theta^{2}+1+\theta x\right)\left(1-e^{-\theta}\right)-\theta e^{-\theta}}{\theta^{2}+1} e^{(-\theta x)}, x=0,1,2,3
\end{gathered}
$$

\section{Probability recurrence relation}

Probability recurrence relation of discrete Shanker distribution may be obtained as

$$
\begin{gathered}
P_{(r+2)}=e^{-\theta}\left(2 P_{r+1}-e^{-\theta} P_{r}\right), r \geq 1 \\
\text { Where } P_{0}=\frac{\left(\theta^{2}+1\right)\left(1-e^{-\theta}\right)-\theta e^{-\theta}}{\theta^{2}+1}, \text { and } \\
P_{1}=\frac{\left(\theta^{2}+1+\theta\right)\left(1-e^{-\theta}\right)-\theta e^{-\theta}}{\theta^{2}+1} e^{-\theta}
\end{gathered}
$$

Volume 5 Issue 4 - 2017

Munindra Borah, Junali Hazarika

Department of Mathematical Sciences, Tezpur University, India

Correspondence: Junali Hazarika, Department of Mathematical Sciences, Tezpur University, Napaam, Tezpur, Assam, India

Received: January 26, 2017 | Published: April 07, 2017

\section{Factorial moment recurrence relation}

Factorial moment generating function (fmgf) may be obtained as

$$
\begin{gathered}
M(t)=G(1+t) \\
=\frac{\left(\theta^{2}+1\right)\left(1-e^{-\theta}\right)-\theta}{\left(\theta^{2}+1\right)\left(1-e^{-\theta}-e^{-\theta} t\right)}+\frac{\theta\left(1-e^{-\theta}\right)}{\left(\theta^{2}+1\right)\left(1-e^{-\theta}-e^{-\theta} t\right)^{2}}
\end{gathered}
$$

The more general form of factorial moment may also be written as

$$
\mu_{[r]}^{\prime}=\frac{r ! e^{-\theta r}\left[\left(\theta^{2}+1\right)\left(1-e^{-\theta}\right)+\theta r\right]}{\left(\theta^{2}+1\right)\left(1-e^{-\theta}\right)^{r+1}}
$$

\section{Size-biased discrete shanker distribution}

If a random variable $X$ have discrete Shanker distribution with parameter $\theta$ then the pmf of the size-biased distribution may be derived as

$$
f_{s}(x ; \theta)=\frac{x P_{x}}{\mu}, x=1,2,3, \ldots
$$

Where $P_{x}$ and $\mu$ denote respectively pmf and the mean of discrete Shanker distribution.

The pmf $f_{s}(x ; \theta)$ of size- biased discrete Shanker distribution with parameters $\theta$ may be derived from (3.1) as

$$
f_{s}(x, \alpha)=\frac{x p_{x}}{\mu}
$$




$$
=x e^{-\theta(x-1)} \frac{\left[\left(\theta^{2}+1+\theta x\right)\left(1-e^{-\theta}\right)-\theta e^{-\theta}\right]\left(1-e^{-\theta}\right)^{2}}{\left[\left(\theta^{2}+1\right)\left(1-e^{-\theta}\right)+\theta\right]} x=1,2,3, \ldots
$$

\section{Recurrence relation of size- biased discrete shanker distribution}

Probability generating function $G(t)$ for Size- biased Discrete Shanker Distribution may be obtained as

$$
\begin{gathered}
G_{s}(t)=\sum_{x=0}^{\infty} t^{x} P_{x} \\
=t \frac{\left[\left(\theta^{2}+1\right)\left(1-e^{-\theta}\right)-\theta e^{-\theta}\right]\left(1-e^{-\theta}\right)^{2}\left(1-e^{-\theta} t\right)+\theta\left(1-e^{-\theta}\right)^{3}\left(1+e^{-\theta} t\right)}{\left[\left(\theta^{2}+1\right)\left(1-e^{-\theta}\right)+\theta\right]\left(1-e^{-\theta} t\right)^{3}}
\end{gathered}
$$

\section{Probability recurrence relation for size- biased discrete shanker distribution}

Probability recurrence relation of Size- biased Discrete Shanker Distribution distribution may be obtained as

$$
P_{r}=e^{-\theta}\left[3 P_{r-1}-3 e^{-\theta} P_{r-2}+e^{-\theta} P_{r-3}\right] \text { for } r>2
$$

where

$$
\begin{gathered}
P_{1}=\frac{\left[\left(\theta^{2}+1+\theta\right)\left(1-e^{-\theta}\right)-\theta e^{-\theta}\right]\left(1-e^{-\theta}\right)^{2}}{\left[\left(\theta^{2}+1\right)\left(1-e^{-\theta}\right)+\theta\right]} \text { and } \\
P_{2}=2 e^{-\frac{\left[\left(\theta^{2}+1+2 \theta\right)\left(1-e^{-\theta}\right)-\theta e^{-\theta}\right]\left(1-e^{-\theta}\right)^{2}}{\left[\left(\theta^{2}+1\right)\left(1-e^{-\theta}\right)+\theta\right]}}
\end{gathered}
$$

\section{Factorial moment recurrence relation for size- biased discrete shanker distribution}

Factorial moment generating function $M_{s}(t)$ of Size- biased discrete Shanker distribution may be obtained as

$$
\begin{gathered}
M_{s}(t)=G_{s}(1+t) \\
\mathrm{M}(\mathrm{t})=(1+t) \frac{\left[\left(\theta^{2}+1\right)\left(1-e^{-\theta}\right)-\theta e^{-\theta}\right]\left(1-e^{-\theta}\right)^{2}\left(1-e^{-\theta}-e^{-\theta} t\right)+\theta\left(1-e^{-\theta}\right)^{3}\left(1+e^{-\theta}+e^{-\theta} t\right)}{\left[\left(\theta^{2}+1\right)\left(1-e^{-\theta}\right)+\theta\right]\left(1-e^{-\theta}-e^{-\theta} t\right)^{3}} \\
\text { More general form } \mu_{[r]}=\frac{r ! e^{-\theta(r-1)}\left[\left(\theta^{2}+1\right)\left(1-e^{-\theta}\right)\left(r+e^{-\theta}\right)+\theta\left(r^{2}-e^{-\theta}\right)\right]}{\left[\left(\theta^{2}+1\right)\left(1-e^{-\theta}\right)+\theta\right]\left(1-e^{-\theta}\right)^{r}}
\end{gathered}
$$

Factorial moment recurrence relation of Size- biased discrete Shanker distribution may be obtained as

$$
\mu_{[r]}^{\prime}=\frac{e^{-\theta}}{A^{3}}\left[3 A^{2} r \mu^{\prime}[r-1]^{-3 A r(}(r-1) e^{-\theta} \mu^{\prime}[r-2]+A r(r-1)(r-2) e^{-2 \theta} \mu^{\prime}[r-3]\right],
$$

Where $A=1-e^{-\theta}$.

$$
\begin{gathered}
\mu_{[1]}=\frac{\left[\left(\theta^{2}+1\right)\left(1+e^{-\theta}\right)+\theta\right]}{\left[\left(\theta^{2}+1\right)\left(1-e^{-\theta}\right)+\theta\right]} \\
\mu_{[2]}=\frac{2 e^{-\theta}\left[\left(\theta^{2}+1\right)\left(1-e^{-\theta}\right)\left(2+e^{-\theta}\right)+\theta\left(4-e^{-\theta}\right)\right]}{\left[\left(\theta^{2}+1\right)\left(1-e^{-\theta}\right)+\theta\right]\left(1-e^{-\theta}\right)^{2}} \\
\mu_{[3]}=\frac{6 e^{-2 \theta}\left[\left(\theta^{2}+1\right)\left(1-e^{-\theta}\right)\left(3+e^{-\theta}\right)+\theta\left(9-e^{-\theta}\right)\right]}{\left[\left(\theta^{2}+1\right)\left(1-e^{-\theta}\right)+\theta\right]\left(1-e^{-\theta}\right)^{3}}
\end{gathered}
$$

\section{Method of estimation of shanker distribution}

The parameter $\theta$ of Shanker distribution has been estimated using Newton's -Raphson method by considering appropriate initial guest value for $\theta$. The function of $\theta$ can be expressed as

\section{Fitting of discrete shanker distribution}

Shanker et al., ${ }^{1}$ fitted Poisson distribution (PD), Poisson- Lindley distribution (PLD) and Poisson-Akash distribution (PAD) to eleven numbers of data sets covering ecology, genetics and thunderstorms. In this investigation discrete Shanker (DS) distribution has been fitted to all 11 data sets have been considered for a comparison (Tables 1-11). ${ }^{2-}$ 
Table I Observed and expected number of homocytometer yeast cell counts per square observed by gosset

\begin{tabular}{|c|c|c|c|c|c|}
\hline \multirow{2}{*}{$\begin{array}{l}\text { No. of yeast cell per } \\
\text { square }\end{array}$} & \multirow{2}{*}{$\begin{array}{l}\text { Observed } \\
\text { Frequency }\end{array}$} & \multicolumn{4}{|c|}{ Expected frequency } \\
\hline & & DS & PD & PLD & PAD \\
\hline 0 & 213 & 213 & 202.1 & 234 & 236.8 \\
\hline I & 128 & 109.15 & 138 & 99.4 & 95.6 \\
\hline 2 & 37 & 47.44 & 47.1 & 40.5 & 39.9 \\
\hline 3 & 18 & 19 & 10.7 & 16 & 16.6 \\
\hline 4 & 3 & 7.25 & 1.8 & 6.2 & 6.7 \\
\hline 5 & I & 2.67 & 0.2 & 2.4 & 2.7 \\
\hline 6 & 0 & 1.48 & 0.1 & 1.5 & 1.7 \\
\hline \multirow[t]{5}{*}{ Total } & 400 & 400 & 400 & 400 & 400 \\
\hline & Estimated $\theta$ & 1.1621 & 0.6825 & 1.950236 & 2.260342 \\
\hline & $\chi^{2}$ & 7.89 & 10.08 & 11.04 & 14.68 \\
\hline & d.f. & 3 & 2 & 2 & 2 \\
\hline & $p$-vale & 0.0468 & 0.0065 & 0.004 & 0.0006 \\
\hline
\end{tabular}

Table 2 Observed and expected number of red mites on apple leaves

\begin{tabular}{|c|c|c|c|c|c|}
\hline \multirow{2}{*}{$\begin{array}{l}\text { No. of yeast cell } \\
\text { per square }\end{array}$} & \multirow{2}{*}{$\begin{array}{l}\text { Observed } \\
\text { Frequency }\end{array}$} & \multicolumn{4}{|c|}{ Expected frequency } \\
\hline & & DS & PD & PLD & PAD \\
\hline 0 & 38 & 38 & 25.3 & 35.8 & 36.3 \\
\hline I & 17 & 22.4 & 29.1 & 20.7 & 20.1 \\
\hline 2 & 10 & 11.02 & 16.7 & II.4 & 11.2 \\
\hline 3 & 9 & 4.97 & 6.4 & 6 & 6.1 \\
\hline 4 & 3 & 2.13 & 1.8 & 3.1 & 3.2 \\
\hline 5 & 2 & 0.88 & 0.4 & 1.6 & 1.6 \\
\hline 6 & I & 0.36 & 0.2 & 0.8 & 0.8 \\
\hline 7 & 0 & 0.14 & 0.1 & 0.6 & 0.7 \\
\hline \multirow[t]{5}{*}{ Total } & 80 & 80 & 80 & 80 & 80 \\
\hline & Estimated $\theta$ & I.0494 & 1.15 & $|.25589|$ & 1.620588 \\
\hline & $\chi^{2}$ & 6.246 & 18.27 & 2.47 & 2.07 \\
\hline & d.f. & 4 & 2 & 3 & 3 \\
\hline & $\mathrm{p}$ - vale & 0.1815 & 0.0001 & 0.4807 & 0.558 \\
\hline
\end{tabular}


Table 3 Observed and expected number of european corn-border of McGuire et al

\begin{tabular}{llllll}
\hline \multirow{2}{*}{$\begin{array}{l}\text { No. of yeast cell } \\
\text { per square }\end{array}$} & Observed & \multicolumn{4}{l}{ Expected frequency } \\
\cline { 2 - 6 } & Frequency & DS & PD & PLD & PAD \\
\hline 0 & 188 & 187.98 & 169.4 & 194 & 196.3 \\
1 & 83 & 85.03 & 109.8 & 79.5 & 76.5 \\
2 & 36 & 33.03 & 35.6 & 31.3 & 30.8 \\
3 & 14 & 11.88 & 7.8 & 12 & 12.4 \\
4 & 2 & 4.07 & 1.2 & 4.5 & 4.9 \\
5 & 1 & 1.99 & 0.2 & 2.7 & 3.1 \\
Total & 324 & 324 & 324 & 324 & 324 \\
& Estimated $\theta$ & 1.2644 & 0.648148 & 2.043252 & 2.345109 \\
& $\chi 2$ & 0.367 & 15.19 & 1.29 & 2.33 \\
& $\chi$ & 3 & 2 & 2 & 2 \\
& d.f. & 0.9470 & 0.0005 & 0.5247 & 0.3119 \\
\hline
\end{tabular}

Table 4 Distribution of number of chromatid aberrations

\begin{tabular}{|c|c|c|c|c|c|}
\hline \multirow{2}{*}{$\begin{array}{l}\text { No. of yeast cell per } \\
\text { square }\end{array}$} & \multirow{2}{*}{$\begin{array}{l}\text { Observed } \\
\text { Frequency }\end{array}$} & \multicolumn{4}{|c|}{ Expected frequency } \\
\hline & & DS & PD & PLD & PAD \\
\hline 0 & 268 & 268 & 231.3 & 257 & 260.4 \\
\hline I & 87 & 87 & 92.8 & 126.7 & 93.4 \\
\hline 2 & 26 & 28.23 & 34.7 & 32.8 & 32.1 \\
\hline 3 & 9 & 8.01 & 6.3 & 11.2 & 11.5 \\
\hline 4 & 4 & 2.18 & 0.8 & 3.8 & 4.1 \\
\hline 5 & 2 & 0.58 & 0.1 & 1.2 & 1.4 \\
\hline 6 & I & 0.15 & 0.1 & 0.4 & 0.5 \\
\hline 7 & 3 & 0.05 & 0.1 & 0.2 & 0.3 \\
\hline \multirow[t]{5}{*}{ Total } & 400 & 400 & 400 & 400 & 400 \\
\hline & $\begin{array}{l}\text { Estimated } \\
\theta\end{array}$ & $\mathrm{I} .4870$ & 0.5475 & 2.380442 & 2.659408 \\
\hline & $\chi^{2}$ & 6.417 & 38.21 & 6.21 & 4.17 \\
\hline & d.f. & 3 & 2 & 3 & 3 \\
\hline & $\mathrm{p}$ - vale & 0.0930 & 0 & 0.1018 & 0.2437 \\
\hline
\end{tabular}

Table 5 Mammalian cytogenetic dosimetry lesions in rabbit lymphoblast induced by streptonigrin (NSC-45383), exposure-60 $\mu \mathrm{g} / \mathrm{kg}$

\begin{tabular}{|c|c|c|c|c|c|}
\hline \multirow{2}{*}{$\begin{array}{l}\text { No. of yeast cell per } \\
\text { square }\end{array}$} & \multirow{2}{*}{$\begin{array}{l}\text { Observed } \\
\text { Frequency }\end{array}$} & \multicolumn{4}{|c|}{ Expected frequency } \\
\hline & & DS & PD & PLD & PAD \\
\hline 0 & 413 & $4 \mid 3.01$ & 374 & 405.7 & 409.5 \\
\hline I & 124 & 134.97 & 177.4 & 133.6 & I 28.7 \\
\hline 2 & 42 & 38.92 & 42.1 & 42.6 & 42.1 \\
\hline 3 & 15 & 10.49 & 6.6 & 13.3 & 13.9 \\
\hline 4 & 5 & 2.71 & 0.8 & 4.1 & 4.6 \\
\hline
\end{tabular}


Table continued.

\begin{tabular}{llllll}
\hline $\begin{array}{l}\text { No. of yeast cell per } \\
\text { square }\end{array}$ & Observed & \multicolumn{2}{l}{ Expected frequency } & \\
\cline { 2 - 6 } & Frequency & DS & PD & PLD & PAD \\
\hline 5 & 0 & 0.68 & 0.1 & 1.2 & 1.5 \\
6 & 2 & 0.22 & 0 & 0.5 & 0.7 \\
Total & 601 & 601 & 601 & 601 & 601 \\
& $\begin{array}{ll}\text { Estimated } \\
\theta\end{array}$ & 1.5385 & 0.47421 & 2.685373 & 2.915059 \\
& $\chi^{2}$ & 5.562 & 48.17 & 1.34 & 0.29 \\
& d.f. & 3 & 2 & 3 & 3 \\
& P- vale & 0.1350 & 0 & 0.7196 & 0.9619 \\
\end{tabular}

Table 6 Mammalian cytogenetic dosimetry lesions in rabbit lymphoblast induced by streptonigrin (NSC-45383), exposure-75 $\mu \mathrm{g} / \mathrm{kg}$

\begin{tabular}{|c|c|c|c|c|c|}
\hline \multirow{2}{*}{$\begin{array}{l}\text { No. of yeast cell per } \\
\text { square }\end{array}$} & \multirow{2}{*}{$\begin{array}{l}\text { Observed } \\
\text { Frequency }\end{array}$} & \multicolumn{4}{|c|}{ Expected frequency } \\
\hline & & DS & PD & PLD & PAD \\
\hline 0 & 200 & 200.01 & 172.5 & 191.8 & 194.1 \\
\hline 1 & 57 & 70.01 & 95.4 & 70.3 & 67.6 \\
\hline 2 & 30 & 21.51 & 26.4 & 24.9 & 24.5 \\
\hline 3 & 7 & 6.17 & 4.9 & 8.6 & 8.9 \\
\hline 4 & 4 & 1.69 & 0.7 & 2.9 & 3.2 \\
\hline 5 & 0 & 0.45 & 0.1 & I & 1.1 \\
\hline 6 & 2 & 0.16 & 0 & 0.5 & 0.6 \\
\hline \multirow[t]{5}{*}{ Total } & 300 & 300 & 300 & 300 & 300 \\
\hline & $\begin{array}{l}\text { Estimated } \\
\theta\end{array}$ & 1.4798 & 0.55333 & 2.35334 & 2.62674 \\
\hline & $\chi^{2}$ & 8.191 & 29.68 & 3.91 & 3.12 \\
\hline & d.f. & 3 & 2 & 2 & 2 \\
\hline & P- vale & 0.0422 & 0 & 0.1415 & 0.2101 \\
\hline
\end{tabular}

Table 7 Mammalian cytogenetic dosimetry lesions in rabbit lymphoblast induced by streptonigrin (NSC-45383), exposure-90 $\mu \mathrm{g} / \mathrm{kg}$

\begin{tabular}{llllll}
\hline \multirow{2}{*}{ No. of yeast cell per square } & Observed & \multicolumn{5}{l}{ Expected frequency } & & \\
\cline { 2 - 6 } & Frequency & DS & PD & PLD & PAD \\
\hline 0 & 155 & 155.01 & 127.8 & 158.3 & 160.7 \\
\hline & 83 & 82.63 & 109 & 77.2 & 74.3 \\
2 & 33 & 37.2 & 46.5 & 35.9 & 35.3 \\
3 & 14 & 15.41 & 13.2 & 16.1 & 16.5 \\
4 & 11 & 6.08 & 2.8 & 7.1 & 7.5 \\
5 & 3 & 2.32 & 0.5 & 3.1 & 3.3 \\
6 & 1 & 1.35 & 0.2 & 2.3 & 2.4 \\
Total & 300 & 300 & 300 & 300 & 300 \\
& & 1.1301 & 0.853333 & 1.617611 & 1.963313 \\
& Estimated $\theta$ & & & & 1.98 \\
& $\chi^{2}$ & 3.432 & 24.97 & 1.51 & 3 \\
& d.f. & 4 & 2 & 3 & 0.5766 \\
\hline
\end{tabular}

Citation: Borah M, Hazarika J. Discrete shanker distribution and its derived distributions. Biom Biostat Int J. 20I7;5(4):I46-I53. 
Table 8 Observed abd expected number of days that experienced $X$ thunderstroms event at cape kennedy, florida for II-year period of record for the month of june, january 1957 to december 1967

\begin{tabular}{llllll}
\hline \multirow{2}{*}{ No. of yeast cell per square } & Observed & \multicolumn{2}{l}{ Expected frequency } & & \\
\cline { 2 - 6 } & Frequency & DS & PD & PLD & PAD \\
\hline 0 & 187 & 187.01 & 155.6 & 185.3 & 187.9 \\
\hline & 77 & 87.72 & 117 & 83.5 & 80.2 \\
2 & 40 & 35.21 & 43.9 & 35.9 & 35.3 \\
3 & 17 & 13.07 & 11 & 15 & 15.4 \\
4 & 6 & 4.62 & 2.1 & 6.1 & 6.6 \\
5 & 2 & 1.58 & 0.3 & 2.5 & 2.7 \\
6 & 1 & 0.79 & 0.1 & 1.7 & 1.9 \\
Total & 330 & 330 & 330 & 330 & 330 \\
& Estimated & 1.2345 & 0.751515 & 1.804268 & 2.139736 \\
& $\chi^{2}$ & & & & 1.35 \\
& $\chi^{2}$ & 3.721 & 31.93 & 1.43 & 3 \\
\hline
\end{tabular}

Table 9 Observed abd expected number of days that experienced $\mathrm{X}$ thunderstroms event at cape kennedy, florida for II-year period of record for the month of july, january 1957 to december 1967

\begin{tabular}{|c|c|c|c|c|c|}
\hline \multirow{2}{*}{ No. of yeast cell per square } & \multirow{2}{*}{$\begin{array}{l}\text { Observed } \\
\text { Frequency }\end{array}$} & \multicolumn{4}{|c|}{ Expected frequency } \\
\hline & & DS & PD & PLD & PAD \\
\hline 0 & 177 & 177.01 & 142.3 & 177.7 & 180 \\
\hline I & 80 & 93.79 & 124.4 & 88 & 84.7 \\
\hline 2 & 47 & 42.01 & 54.3 & 41.5 & 40.9 \\
\hline 3 & 26 & 17.32 & 15.8 & 18.9 & 19.4 \\
\hline 4 & 9 & 7.79 & 3.5 & 8.4 & 8.9 \\
\hline 5 & 2 & 3.08 & 0.7 & 6.5 & 7.1 \\
\hline \multirow[t]{5}{*}{ Total } & 341 & 341 & 341 & 341 & 341 \\
\hline & $\begin{array}{l}\text { Estimated } \\
\theta\end{array}$ & 1.1348 & 0.8739 & 1.583536 & 1.938989 \\
\hline & $\chi^{2}$ & 6.972 & 39.74 & 5.15 & 5.02 \\
\hline & d.f. & 4 & 2 & 3 & 3 \\
\hline & $\mathrm{p}$ - vale & 0.1374 & 0 & 0.1611 & 0.1703 \\
\hline
\end{tabular}

Table 10 Observed abd expected number of days that experienced $X$ thunderstroms event at cape kennedy, florida for II-year period of record for the month of august, january 1957 to december 1967

\begin{tabular}{|c|c|c|c|c|c|}
\hline \multirow{2}{*}{ No. of yeast cell per square } & \multirow{2}{*}{$\begin{array}{l}\text { Observed } \\
\text { Frequency }\end{array}$} & \multicolumn{4}{|c|}{ Expected frequency } \\
\hline & & DS & PD & PLD & PAD \\
\hline 0 & 185 & 184.99 & 151.8 & 184.8 & 187.5 \\
\hline I & 89 & 92.42 & 122.9 & 87.2 & 83.9 \\
\hline 2 & 30 & 39.27 & 49.7 & 39.3 & 38.6 \\
\hline 3 & 24 & 15.39 & 13.4 & 17.1 & 17.5 \\
\hline 4 & 10 & 6.74 & 2.7 & 7.3 & 7.6 \\
\hline 5 & 3 & 2.19 & 0.5 & 5.3 & 5.9 \\
\hline \multirow[t]{5}{*}{ Total } & $34 I$ & $34 I$ & 341 & $34 I$ & 341 \\
\hline & $\begin{array}{l}\text { Estimated } \\
\theta\end{array}$ & I.I828 & 0.809384 & 1.693425 & 2.038417 \\
\hline & $\chi^{2}$ & 8.987 & 49.49 & 5.03 & 4.69 \\
\hline & d.f. & 4 & 2 & 3 & 3 \\
\hline & $\mathrm{p}$ - vale & 0.0414 & 0 & 0.1696 & 0.196 \\
\hline
\end{tabular}

Citation: Borah M, Hazarika J. Discrete shanker distribution and its derived distributions. Biom Biostat Int J. 20I7;5(4): I46-I53.

DOI: I0.I5406/bbij.20I7.05.00I40 
Table II Observed abd expected number of days that experienced $X$ thunderstroms event at cape kennedy, florida for II-year period of record for summesr, january 1957 to december 1967

\begin{tabular}{llllll}
\hline \multirow{2}{*}{ No. of yeast cell per square } & Observed & \multicolumn{2}{l}{ Expected frequency } & & \\
\cline { 2 - 6 } & Frequency & DS & PD & PLD & PAD \\
\hline 0 & 549 & 549.01 & 449 & 547.5 & 555.1 \\
1 & 246 & 274.27 & 364.8 & 259 & 249.2 \\
2 & 117 & 117 & 116.54 & 148.2 & 116.9 \\
3 & 67 & 45.67 & 40.1 & 51.2 & 52.3 \\
4 & 25 & 17.04 & 8.1 & 21.9 & 23.2 \\
5 & 7 & 7.16 & 1.3 & 9.2 & 10 \\
6 & 1 & 2.31 & 0.5 & 6.3 & 7.3 \\
Total & 1012 & 1012 & 1012 & 1012 & 1012 \\
& Estimated & 1.1828 & 0.812253 & 1.68899 & 2.033715 \\
& $\theta$ & 16.824 & 119.45 & 9.6 & 9.4 \\
& $\chi 2$ & 5 & 3 & 4 & 4 \\
& d.f. & 0.0048 & 0 & 0.0477 & 0.0518 \\
\hline
\end{tabular}

\section{Conclusions}

In this article, the discrete Shanker distribution has been introduced by discretizing the continuous Shanker distribution. We have studied some properties of the distributions. Further the applications of the distribution and goodness of fit of the distribution.

\section{Acknowledgement}

None.

\section{Conflict of interest}

None.

\section{References}

1. Shanker R. Amarendra distribution and its applications. American journal of Mathematics and Statistics. 2016;6(1):44-56.

2. Adhikari TR, Srivastava RS. A size-biased poisson-lindley distribution. International Journal of Mathematical Modeling and Physical Sciences. 2013;1(3):1-5.

3. Adhikari TR, Srivastava RS. Poisson-size-biased lindley distribution International Journal of Scientific and Research Publication. 2014;4(3):1-6.

4. Borah M.The Genenbauer distribution revisited: Some recurrence relation for moments, cumulants, etc., estimation of parameters and its goodness of fit. Journal of Indian Society of Agricultural Statistics. 1984;36(1):7278.

5. Borah M, Begum R. Some properties of Poisson-Lindley and its derive distributions. Journal of the Indian Statistical Association. 2000;4:13-25.

6. Borah M, Deka Nath A. A study on the inflated poisson lindley distribution Journal of Indian Soc. Ag. Statistics. 2001;54(3) 317-323.

7. Dutta P, Bora M. Some properties and application of size-biased poissonlindley distribution. International Journal of Mathematical Archive. 2014a;5(1):89-96.

8. Dutta P, Borah M. Zero-modified poisson-lindley distribution. Mathematics and Computers in Simulation. (2014b);79(3):279-287.

9. Fisher RA. The effects of methods of ascertainment upon the estimation of frequency. Ann Eugenics. 1934;6(1):13-25.

10. Ghitany ME, Atiech B, Nadarajah S. Lindley distribution and its applications, Mathematics and Computers in Simulation. (2008a);78(4):493-506.

11. Ghitany ME, Al-Mutari DK. Size-biased Poisson - lindley distribution and its application. Metron-International Journal of Statistics LXVI. 2008b;(3):299-311.

12. Ghitany ME, Al-Mutairi DK. Estimation methods for the discrete Poisson-Lindley distribution. J Stat Comput Simul. 2009;79(1) 1-9.

13. Gomez-Deniz E. Another generalization of the geometric distribution. Test. 2010;19(2):399-415.

14. Gove HJ. Estimation and application of size-biased distributions in forestry. In: Amaro \& D. Reed, editors. Modeling Forest systems A. CAB International, Wallingford, UK. 2003;201-212.

15. Johnson NL, Kemp AW, Kotz S. Univariate discrete distributions. Hoboken NJ and John Wiley \& Sons, USA; 2005.

16. Kemp AW. The discrete half-normal distribution. MA Advances in Mathematical and Statistical Modelling. Birkhauser, Boston, USA; 2008:353-365.

17. Krishna H, Pundir PS. Discrete Burr and discrete pareto distributions. Stat Methodol. 2009;6:177-188.

18. Lappi J, Bailey RL. Estimation of diameter increment function or other relations using angle-count samples. Forest Science. 1987;33:725-739. 
19. Lindley DV. Fiducial distributions and Bayes' theorem. Journal of the Royal Statistical Society, Series B. 1958;20(1):102-107.

20. Mahmoudi E, Zakerzadeh H. Generalized poisson lindley distribution. Communications in Statistics-Theory and Methods. 2010; 39(10):17851798.

21. Marshall AW, Olkin I. A new method for adding a parameter to a family of distributions with application to the exponential and Weibull families. Biometrika. 1997;84(3):641-652.

22. Nakagawa T, Osaki S.The discrete Weibull distribution. IEE Trans Reliab. 1975;24(5):300-301.

23. Patil GP, Ord JK. On size-biased sampling and related form-invariant weighted distributions. Sankhya. 1975;38(1):48-61.

24. Patil GP, Rao CR. The weighted distributions: a survey and their applications. In Applications of Statistics. 1977;383-405.

25. Patil GP, Rao CR. Weighted distributions and size-biased sampling with applications to wild life populations and human families. Biometrics. 1978;34:179-189.

26. Rao CR. On discrete distributions arising out of methods on ascertainment, classical and contagious discrete distribution. In: Patil GP, editor. Statistical Publishing Society. 1965;320-332.

27. Roy D. Discrete rayleigh distribution. IEEE Trans. Reliab 2004;53(2):255-260
28. Sankaran M. The discrete poisson-lindley distribution. Biometrics. 1970;26:145-149.

29. Shanker R, Mishra A. A two parameter poisson- lindley distribution. International journal of Statistics and Systems. 2014;9(1): 79-85.

30. Shanker R. Shanker distribution and its Applications. International Journal of Statistics and Applications. 2015;5(6):338-348.

31. Shanker R, Mishra A. A quasi Lindley distribution. African Journal of Mathematics and Computer Science Research. 2013; 6(4):64-71.

32. Shanke R, Sharma S, Shanker R. A discrete two-parameter poissonlindley distribution. Journal of the Ethiopian Statistical Association. 2012;21:15-22.

33. Shanke R, Sharma S, Shanker R. Janardan distribution and its applications to waiting times data. Indian Journal of Applied research . 2013;3(8):500 502 .

34. Shanker R, Tekie Asehun Leonida. A new quasi poisson- lindley distribution. International Journal of Statistics and Systems. 2014;9(1):87-94.

35. Shanke, R, Sharma S, Shanker R, et al. The discrete Poisson- Janardan distribution with applications. International Journal of Soft Computing and Engineering. 2014;4(2):31-33.

36. Van Deusen PC. Fitting assumed distributions to horizontal point sample Diameters. Forest Science. 1986;32(1):146-148. 